\title{
Políticas sociales, instituciones, prácticas profesionales
}

\author{
Elizabeth Ortega Cerchiaro
}

\section{SciELO Books / SciELO Livros / SciELO Libros}

CERCHIARO, E. O. Políticas sociales, instituciones, prácticas profesionales. In: COSTA, L. C., NOGUEIRA, V. M. R., and SILVA, V. R., orgs. A política social na América do Sul: perspectivas e desafios no século XXI [online]. Ponta Grossa: Editora UEPG, 2013, pp. 247-258. ISBN 978-857798-231-8. Available from: doi: 10.7476/9788577982318.0012. Also available in ePUB from: http://books.scielo.org/id/rfv9p/epub/costa-9788577982318.epub.

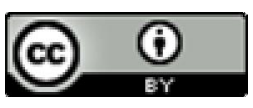

All the contents of this work, except where otherwise noted, is licensed under a Creative Commons Attribution $\underline{4.0 \text { International license. }}$

Todo o conteúdo deste trabalho, exceto quando houver ressalva, é publicado sob a licença Creative Commons Atribição 4.0.

Todo el contenido de esta obra, excepto donde se indique lo contrario, está bajo licencia de la licencia Creative Commons Reconocimento 4.0. 


\section{Políticas sociales, instituciones, prácticas profesionales}

Elizabeth Ortega Cerchiaro

\section{Introducción}

La reflexión acerca de los sistemas de protección social y de la implementación de políticas sociales en los diferentes momentos históricos permite analizar la complejidad de las modalidades de intervención en lo social que se ven asociadas a ellas.

En el presente artículo se pretende, en primer lugar, realizar un breve análisis socio histórico del denominado sistema de bienestar instalado tempranamente en Uruguay, sus modificaciones a lo largo de los siglos XX y XXI y, a partir de ello, focalizar la atención en los diferentes dispositivos de intervención social, particularmente los relacionados con el Trabajo Social como profesión.

Se trata, fundamentalmente, de identificar ciertos trazos en esas modalidades de intervención, asociados a los procesos de individualización de la vida social, que se pueden considerar como elementos de permanencia en el largo plazo y que adquieren formatos renovados en los diferentes momentos históricos. Se analizará particularmente cómo se producen, en el campo médico sanitario (de la mano del higienismo y del preventivismo, de principios y de mediados del siglo XX, asociados genéticamente al nacimiento y primeros desarrollos del Trabajo Social en Uruguay) ciertos cambios en las percepciones de la génesis de las enfermedades, que son transportados al campo de lo social, lo cual colabora en otorgar fundamentos a los procesos de responsabilización del individuo y de la familia acerca de problemas que anteriormente eran considerados como de responsabilidad y de respuesta social. Se destaca en ese proceso el papel central de las instituciones.

\section{Una mirada socio histórica al sistema de protección social en Uruguay}

El denominado sistema de bienestar en Uruguay tuvo un temprano desarrollo, con una expansión importante ya en las primeras tres décadas del siglo XX. ${ }^{1}$ Las características peculiares de la formación social uruguaya,

\footnotetext{
1 Período que es conocido como del Primer Batllismo, debido al protagonismo del presidente José Batlle y Ordóñez que ejerció la Presidencia de la República en dos períodos en esos treinta años.
} 


\section{Elizabeth Ortega Cerchiaro}

respecto del temprano proceso de secularización, la tardía hegemonía de la fuerza del Estado en todo el territorio nacional y la temprana extensión de los derechos sociales, así como la fortaleza de los partidos políticos, trazaron líneas de larga duración que atravesaron todo el siglo XX (CAETANO; RILLA, 1996).

Filgueira (1994), destaca la implantación de un cúmulo de políticas sociales que se apoyaron en una retórica universalista desde el punto de vista de su formulación a nivel genérico y en su base jurídica. Ese Estado interventor en el diseño de las políticas sociales se estructuraba sobre cuatro pilares que Filgueira (1994) identifica, en primer lugar, con las leyes y resoluciones del Ejecutivo concernientes al funcionamiento del mercado laboral (jornada laboral de ocho horas, semana laboral de seis días, regulación del trabajo femenino e infantil, legislación sobre accidentes de trabajo, etcétera). El segundo pilar, se refiere a la política de retiro de la fuerza de trabajo, que se fue haciendo a lo largo del período cada vez más abarcativa. El tercer pilar, es la instrucción pública, que se comienza a consolidar hacia fines del siglo XIX y avanza en el siglo XX con la instalación de la enseñanza secundaria y la reformulación de la universitaria. El cuarto pilar, tiene relación con un cambio sustancial en la consideración del papel del Estado en la administración de las instalaciones de beneficencia existentes que atendían los aspectos relacionados con la salud pública.

Estas políticas se enmarcaron en el proceso de consolidación del sistema democrático, en que el papel de los partidos políticos fue central con una cultura política fuertemente estatalista. Castellanos (1996) destaca que las tres primeras décadas del siglo XX representan años constitutivos del proceso de extensión de derechos políticos y sociales, del sistema que denomina de "bienestar de partidos", debido a la fortaleza de esos agentes en el contexto del afianzamiento del Estado uruguayo.

Se destaca, en el período posterior a la Segunda Guerra Mundial, el afianzamiento de políticas sociales de carácter universalista, en las que se mantienen los cuatro pilares anteriormente señalados por Filgueira, como base del sistema de bienestar en Uruguay, en el marco de una sociedad que buscaba en el sistema de sustitución de importaciones una salida económica exitosa y sustentable.

A partir de mediados de la década de 1940, el advenimiento del denominado neobatllismo (D'ELÍA, 1982) marca un proceso de continuidades con el batllismo temprano: consolidación de los derechos sociales, protagonismo de los nucleamientos partidarios, y aumento considerable de la mano de obra empleada, con un papel muy relevante de las organizaciones sindicales en ese proceso. 
Hacia mediados de la década de 1950 ese modelo comienza a dar indicios de decadencia y se inicia un proceso en que:

Una sociedad amortiguadora, hiperintegrada, partidocrática, frágil/ próspera, pero próspera al fin, declinó en su capacidad de innovación y reproducción, para ingresar en una fase de radicalizaciones y violencia sin precedentes en el siglo XX. (CAETANO; RILLA, 1996, p.198).

Ese proceso estuvo enmarcado en un contexto internacional que dejó de ser favorable para la economía uruguaya. La crisis económica que comienza a manifestarse desde mediados de los años cincuenta fue reforzada por otros factores: cambio radical en los mercados capitalistas, disminución de la demanda internacional de productos latinoamericanos, disminución de los precios internacionales de las materias primas, lo que implicó un aumento del endeudamiento. Pero, también tuvieron incidencia otros problemas de orden estructural de vieja data: el estancamiento ganadero que la crisis vino a ratificar y profundizar, volvió a reconocer razones de larga duración, sumándose el freno de la expansión agrícola y la caída del proceso de sustitución de importaciones.

La bibliografía consultada (ASTORI, 2005; FINCH, 1980) coincide en afirmar que el carácter estructural de la crisis se refería a una modificación radical en el mercado mundial, en la inserción internacional del país y en la quiebra definitiva de la construcción económica de larga duración que había demostrado capacidad de ajuste ante coyunturas adversas. Y comienza en forma más notoria la lucha por la apropiación de un excedente económico estancado: especulación, y corporativización de las relaciones políticas y sociales que se manifiesta en el descontento de los ganaderos, de los industriales, y de los trabajadores.

Este complejo proceso condujo, a partir del Golpe de Estado de 1973 a la instalación de una dictadura que se extenderá hasta 1985. A pesar de las consecuencias nefastas en cuanto a la instalación de dispositivos de represión inéditos en la historia de Uruguay, según Midaglia y Antía (2007) en este período, no se revirtió la matriz de bienestar, aunque sí se resintió la calidad de los servicios, se deterioraron las prestaciones y hubo restricciones del gasto público.

En el período del primer gobierno democrático, que se instala en 1985, se produjo una serie importante de cambios en las políticas sociales que retomaron en muchos de sus aspectos la matriz instalada desde principios del siglo XX.

Sin embargo, es a partir de la década de 1990, cuando se produce una serie de reformas en el marco de una estrategia de desarrollo con orientación al mercado, que apuntan a la desregulación laboral, a reformar el sistema jubilatorio, el sistema educativo (que, a pesar de ello mantuvo su orientación estatista y universalista), a instalar nuevos programas sociales 


\section{Elizabeth Ortega Cerchiaro}

estatales que pasan a ser implementados por asociaciones de la sociedad civil, Organizaciones No Gubernamentales, dirigidos a poblaciones vulnerables, y a implementar prestaciones que son cada vez más focalizadas (MIDAGLIA; ANTÍA, 2007).

En forma coincidente Serna (2008) señala como características generales del sistema instalado en la década de 1990, la reducción del papel del Estado, la transitoriedad de los programas sociales, y la privatización o semiprivatización de la implementación de las políticas sociales. De acuerdo con su caracterización del período, las que denomina "redes sociales de seguridad" que son instaladas en esa década, constituyeron una estrategia de compensación y contención hacia los sectores más excluidos, focalizada en la extrema pobreza. Destaca, también, la responsabilización a la sociedad y a la familia respecto de cuestiones que anteriormente eran atribuidas a la sociedad o al Estado

Hobsbawm (2007, p.336), alude a los cambios consolidados hacia las décadas del 1980 y 1990 como

[...] el triunfo del individuo sobre la sociedad, o mejor, como la ruptura de los hilos que hasta entonces habían imbricado a los individuos en el tejido social. Y es que este tejido no sólo estaba compuesto por las relaciones reales entre los seres humanos y sus formas de organización, sino también por los modelos generales de esas relaciones y por las pautas de conducta que era de prever que siguiesen en su trato mutuo los individuos, cuyos papeles estaban predeterminados, aunque no siempre escritos.

Los cambios que se producen en los comienzos del siglo XXI están marcados por la instalación de la grave crisis económica que eclosiona en 2002 en Uruguay, a partir de la cual se producen procesos de empobrecimiento y de fragmentación social, con una tendencia concentradora en la distribución del ingreso

El período que se inicia en 2005 con la asunción del Frente Amplio al gobierno, puede definirse por una serie de medidas que afectan el sistema de protección social en Uruguay. Ellas se pueden caracterizar, siguiendo a Midaglia y Antía (2007) como de enfrentamiento al modelo implementado en las décadas anteriores que había implicado apertura económica, liberalización financiera, reducción del rol del Estado y reformas pro mercado. Por otro lado, las autoras destacan la recuperación del Estado en términos de intervención en la vida social, económica y política, la voluntad de incidir en la estructura de desigualdad y de aliviar las situaciones de pobreza más acuciantes.

Por su parte, Serna (2008, p.5) coloca como características comunes de las que denomina "redes de protección social" que son instaladas a partir de 2005, con las predominantes en la década de 1990, que tienen como 
objetivo prioritario: "aliviar las condiciones de carencia social extrema en tanto respuesta a las consecuencias sociales regresivas de la crisis económica y la exclusión laboral del mercado de trabajo"; la focalización en sectores de extrema pobreza, políticas descentralizadas en el territorio, participación de la sociedad civil; estrategias de creación de empleos "mínimos" protegidos y un énfasis colocado en la reintegración al sistema educativo.

Asímismo, Serna (2008) indica como elementos de innovación de las redes de protección social un freno a las estrategias de privatización de las políticas sociales, una creciente valoración del sector público social, el enfoque desde los derechos ciudadanos, los mecanismos de transferencia de ingresos monetarios y la instalación de contrapartidas por parte de las familias.

Como síntesis, Serna (2008) a firma que el costo global de los programas sociales de transferencia de renta es marginal, con un escaso impacto en la reducción de la desigualdad económica, en que el efecto sobre la indigencia y la pobreza extrema es el más notorio.

El breve análisis socio histórico del sistema de protección social instalado en Uruguay desde principios del siglo XX permite reflexionar, en el punto siguiente, acerca de las diferentes modalidades de intervención en lo social que predominaron a lo largo del período considerado.

\section{Las viejas y nuevas modalidades de intervención en lo social ${ }^{2}$}

Es posible afirmar que el Estado batllista, instalado en las primeras tres décadas del siglo XX, se fue apropiando de espacios que eran anteriormente ocupados por agentes asociados a las organizaciones de caridad y filantropía en las actividades de atención e instrumentación de prestaciones sociales a las familias pobres, y fue construyendo, también, iniciativas de profesionalización de esas actividades, siendo las primeras conocidas, aquellas asociadas al higienismo novecentista (ACOSTA, 1997; ORTEGA, 2008).

A pesar de ello, existía la preocupación permanente por distinguir de alguna manera "científica" al "meritorio del falso pobre", como forma de definir si la ayuda era consignada o negada. Este lugar del hacedor de esa discriminación,

\footnotetext{
2 Este ítem fue elaborado tomando como base la investigación desarrollada en el marco de la Maestría en Servicio Social, cuya tesis fuera publicada en Ortega, E. 2008 El Servicio Social y los procesos de medicalización de la sociedad uruguaya en el período neobatllista. Montevideo: Trilce y en la tesis de Doctorado: Ortega, E. "Medicina, religión y gestión de lo social. Un análisis genealógico de las transformaciones del Servicio Social en el Uruguay. 1955-1973". Tesis defendida en 2009 como requisito para la obtención del título de Doctora en Ciencias Sociales con especialización en Trabajo Social, Facultad de Ciencias Sociales, Universidad de la República, Uruguay. Orientadora Prof. Dra. Myriam Mitjavila. En prensa.
} 


\section{Elizabeth Ortega Cerchiaro}

según Morás (2000, p.50), se fue corriendo tempranamente del campo religioso al campo profesional, y resaltando el carácter moral de la asistencia.

Éstos como representantes de nuevas formas de conocimiento científico, tienen un multifacético rol: tanto contribuirán al disciplinamiento como a la despersonalización y exigencia de obligatoriedad de la asistencia. [...] las incipientes ciencias sociales intentan ser el instrumento para una categorización racional de las diversas situaciones planteadas.

A las estrategias higienistas de intervención en lo social asentadas en modelos etiológicos exógenos, que en nombre de la salud tenían un fuerte componente disciplinador, es posible asociar el surgimiento de profesiones que, como el Trabajo Social, nacen subordinadas a la profesión médica. ${ }^{3}$ Ello se tradujo en la atribución social a la profesión incipiente, de una serie de funciones sociales caracterizadas por su carácter inspectivo y de control, especialmente dirigidas a las familias pobres.

Durante el pasaje del siglo XIX al XX, se produce una extensión de aquellas concepciones que atribuyen al medio una fuerte influencia en el origen de las condiciones sanitarias de la población, siendo el higienismo su soporte institucional instalado en la sociedad uruguaya, a través de dispositivos que formaban parte de dos de los sistemas más fuertemente integradores: la educación y la salud, siendo los médicos higienistas agentes relevantes en ese diagrama de elementos de carácter disciplinador, en el sentido que Foucault $(1998,2004,2006)$ atribuye a ese término.

Hacia mediados de siglo XX se produce una inflexión en los modos de intervención en lo social, particularmente en el ámbito de la salud, asociada a la instalación del modelo preventivista que, al apoyarse en modelos etiológicos endógenos, transporta un cambio fundamental en la consideración del papel del individuo en las formas de gestión de lo social. Dicha inflexión estuvo asociada, en el caso uruguayo, a "cambios en la institucionalidad, con el surgimiento de nuevos espacios organizacionales de atención a la salud, la formación de nuevos agentes sanitarios, la influencia de organismos internacionales en los modelos de gestión en el campo de la salud" y en el papel de las diferentes categorías profesionales, entre las cuales se encuentra el Trabajo Social (ORTEGA, 2008, p.126).

Podría decirse que las propuestas y contenidos presentes en las intervenciones preventivistas en el ámbito de la salud constituyen una de las manifestaciones incipientes que los procesos de individualización social adoptan en la vida contemporánea (ORTEGA; VECINDAY, 2009).

Véase: Kruse (1987, 1994), Bralich (1993), Acosta (1997), Ortega (2008). 
Mitjavila y Da Silva (2004, p.70) indican que "la individualización se refiere a los mecanismos y procesos que tornan la percepción de los problemas sociales como problemas individuales en función de disposiciones psicológicas y familiares". Esto es, en otras palabras, que hacia mediados del siglo XX, la inflexión en los modelos etiológicos hacia modalidades de explicación de los problemas sociales como manifestaciones de problemas ocasionados en decisiones individuales, tiene un fuerte impacto en la transformación de los dispositivos de intervención en lo social.

Es posible afirmar, entonces, que a lo largo de la segunda mitad del siglo $\mathrm{XX}$, se consolida el pasaje hacia un modelo de abordaje de las problemáticas sociales de carácter endógeno, que estaría asociado a la responsabilización del individuo o de la familia por procesos que, anteriormente, eran considerados como de respuesta o responsabilidad de la sociedad.

Es posible decir, que hacia el final del período considerado, ese proceso cobra fuerza en función de un cada vez más notorio pasaje del escenario donde prevalecían políticas de integración a otro donde, también paulatinamente, se pueden identificar elementos propios de las políticas de inserción, de acuerdo con la expresión de Castel (2004).

Desde el punto de vista del Trabajo Social, varios son los procesos que se suman, o se complementan en el sentido de su proceso de profesionalización: uno es la ampliación de los requerimientos dirigidos al Servicio Social en las organizaciones tanto estatales como privadas, trascendiendo los ámbitos de la salud y la educación, pero manteniendo una modalidad "preventivista". Recordemos que dicha modalidad se pudo identificar como una incipiente expresión de los procesos de individualización de la vida social.

La ampliación de los requerimientos hacia la profesión fue posible porque, cualquiera fuera el campo de actuación del Trabajo Social, las expectativas sobre el papel de esta profesión se concentraban cada vez más en la selección de aquellos que recibirían prestaciones sociales, en un contexto de recursos cada vez más escasos. En ese sentido, comienzan a hacerse visibles dos sustratos institucionales de esa demanda para el Trabajo Social: la focalización en la pobreza y la necesidad cada vez más presente de categorizar a la población que demandaba su acceso a prestaciones sociales.

En las últimas décadas del siglo XX y en el pasaje al XXI la intervención del Trabajo Social ha estado asociada a la progresiva estructuración de nuevos sistemas de percepción y categorización de lo social que han permeado aspectos cada vez más amplios de las relaciones entre estructuras y agentes sociales.

Es en ese sentido, que se plantean demandas institucionales hacia la profesión que están permeadas por esas estrategias individualizadoras que se encuentran presentes en la vida social. 


\section{Elizabeth Ortega Cerchiaro}

Las instituciones son, justamente, las capaces de naturalizar esas formas de mirar, otorgándoles, como dice Douglas (1996), un fundamento en la "razón o en la naturaleza". Las instituciones como conjuntos de normas, valores, papeles, costumbres, elementos simbólicos se imponen y son responsables de la construcción de lo social, conforman la columna vertebral de lo moral en una sociedad y, en ese sentido, también los principios de clasificación, tienden a soportar y a soportarse en las instituciones.

La autora destaca el dominio de las instituciones sobre los procesos de clasificación y reconocimiento y cómo de ellas depende la conformación del pensamiento de los individuos, siendo la sociedad la que le da al individuo las clasificaciones, las operaciones lógicas, "las metáforas orientadoras" ya que: "para adquirir legitimidad cualquier tipo de institución necesita una fórmula que fundamente su bondad en la razón o en la naturaleza". (DOUGLAS, 1996, p.74).

Afirma, también, que la institución es tan sólo una convención, de ahí que la defina como una "agrupación social legitimada": "[...] se parte aquí de la base de que, en caso de amenaza la mayoría de las instituciones establecidas son capaces de basar sus pretensiones de legitimidad en su encaje en la naturaleza del universo". (DOUGLAS, 1996, p.75)

Incluso, según Douglas (1996, p.1 44), la actividad de construcción de los tiempos pasados está influida por el presente y es regida por las instituciones y, en ese sentido, ellas organizan la memoria pública: "El gran triunfo del pensamiento institucional consiste en conseguir que las instituciones sean completamente invisibles".

En un sentido que se puede considerar complementario, Grassi (2007, p.31) indica:

Lo problemático y lo que tiene consecuencias en la disputa (política) por la representación del mundo social es la naturalización de las clasificaciones y de los atributos que le sirven de variables, porque en ese movimiento se pierde de vista el proceso sociohistórico y las relaciones por las cuales los diferentes grupos se distribuyen de manera diferencial y desigual en el espacio social, así como las carencias y las cualidades, los recursos de la política y las responsabilidades por los problemas. Cuando esto se pierde de vista, el relevamiento de las carencias deviene confirmatorio de las estructuras, y las categorías que designan grupos de población, entran al discurso técnico político como si constituyeran grupos sociales espontáneamente delimitados.

La visualización del espacio de intervención social como un ámbito neutro otorga la atribución de neutralidad a las propias intervenciones profesionales. Este proceso se puede asociar con el planteo de Douglas, respecto al 
proceso de "naturalización" de las relaciones sociales que se llevan a cabo en y a través de las instituciones.

Weisshaupt (1988, p.54), por su parte, realiza un aporte novedoso en ese aspecto, describiendo el doble movimiento de los procesos de institucionalización: en primer lugar, el de la propia profesión, que se lleva a cabo a través de varios mecanismos, como son la fragmentación temática, o sea el abordaje de diferentes problemáticas sociales como la salud, la educación, etcétera, con una lógica segmentada, y la tendencia a la "individualización de los problemas, a considerar los mismos bajo una perspectiva de patologías, ya que los patrones de normalidad funcionan como modelos de referencia [...]"4. En segundo lugar, el autor identifica aquel movimiento que involucra a las "poblaciones asistidas" y que persigue como objetivo su institucionalización. La hipótesis que guía el trabajo de Weisshaupt (1988, p.123) plantea, justamente, que "la carencia de institucionalización o los riesgos de desinstitucionalización o de institucionalización indebida constituirían el objeto básico de la práctica del servicio social".

Con relación a participación de las profesiones en los procesos de individualización social, De Swaan (1992) aporta el punto de vista de cómo los individuos orientan cada vez más su vida diaria hacia las actitudes y nociones que los profesionales difunden, y adoptan modelos de conducta en consonancia.

\section{Reflexiones finales}

Interesa subrayar, para los fines del presente trabajo, entonces, la importancia que asumen las instituciones en la relación entre individuo y sociedad, como lugar donde se construyen y reconstruyen las relaciones de poder y de saber que constituyen las bases institucionales de los procesos de profesionalización de ciertas ocupaciones, entre otra, la del Trabajo Social.

En ese caso, la profesión ocupa un lugar especialmente acondicionado por la instalación de un sistema de percepción sobre la necesidad de que ciertos aspectos de la vida social pasen a ser objeto de su intervención: lo que se destaca es el vínculo con los individuos que son clasificados, categorizados, y los efectos que ello produce en sus comportamientos. Los profesionales aplican ciertos conocimientos, los validan, trabajan en el seno de instituciones que garantizan su legitimidad, e integran los mecanismos que hacen posible que las clasificaciones interactúen con los individuos (HACKING, 2001).

4 Traducción propia. 
La necesidad de clasificar, de cuantificar, de distinguir, de identificar, de focalizar, se han transformado en parte fundamental de los discursos y de las prácticas de la profesión. Así como un discurso que pone el énfasis en la importancia de las decisiones individuales sobre las colectivas (como es el caso del denominado emprendedurismo); los componentes asociados a los procesos de toma de decisión autónoma de los individuos, cuando los soportes sociales construidos previamente ya no existen.

Se produce una tecnificación creciente en la gestión de lo social, en que el manejo de sistemas informáticos permite la creación de campos documentales cada vez más sofisticados.

Las estrategias de intervención basadas en formas de gestión de lo social asociadas a las estrategias preventivistas hacen especial hincapié en la educación, en el fomento de comportamientos basados en el autocuidado y en la difusión de estilos de vida que sean "saludables".

Para ese tipo de estrategias los profesionales se deben dotar de herramientas que permitan la implantación de estrategias de capacitación, promoción de estilos de vida saludables, frente a un proceso en el cual, las prestaciones aparecen asociadas a cálculos cada vez más precisos y sofisticados de detección de las carencias.

Aparecen en los discursos profesionales términos como empoderamiento, resiliencia que, se entiende, pueden llegar a formar parte de los dispositivos de intervención fuertemente individualizadores de la vida social, en esta primera década del siglo XXI.

En la senda de dichos cambios, los procesos de individualización de la vida social están genéticamente asociados a las formas instaladas de clasificación de las personas que determinan las modalidades de intervención. Existiría una intrincada conexión entre los procesos individualizadores y las formas de clasificar a las personas, siendo que el principal nexo sería la consideración de su "normalidad" o su "anormalidad" y, además, de si es capaz de hacerse cargo de sí mismo y, por extensión, de su familia.

La atribución al individuo y a la familia de responsabilidad en la génesis de los problemas sociales que les afectan, se ve muchas veces fundamentada en la consideración de las carencias en los conocimientos adquiridos por ellos mismos, lo cual les impediría escoger las mejores opciones posibles en términos de "estilos de vida". A partir de esa consideración, es que las estrategias de intervención de las profesiones, en particular el Trabajo Social, pasan a tener centralmente un carácter educativo que, se supone, contribuiría a mejorar las posibilidades de opción por formas más exitosas de autocuidado.

Desde esta perspectiva, los aspectos más relevantes examinados, se traducirían en que, a partir del impacto de las corrientes preventivistas, que 
comenzaron a ubicar al individuo y a la familia como focos en la atribución de responsabilidades por cuestiones que anteriormente habían sido, en forma predominante, adjudicadas al medio externo, los discursos y las prácticas profesionales comienzan a dirigirse hacia las propias conductas de los individuos, sus hábitos y estilos de vida. Dichas corrientes preventivistas sostienen que, a través de estrategias de intervención de cuño educativo, es posible "prevenir" consecuencias que, se asegura, serán negativas.

Los nuevos discursos tendientes a favorecer, por ejemplo, estilos de vida saludable, en la mayor parte de los casos son parte de intervenciones dirigidas a esos mismos individuos y familias que ya han perdido, en términos de Castel (2004), los soportes que les permitirían pensar en el futuro como posible.

\section{Bibliografía}

ACOSTA, L. Modernidad y servicio social: un estudio sobre la génesis del Servicio Social en el Uruguay. Disertación (Maestría en Servicio Social) Rio de Janeiro: UFRJ, 1997.

ASTORI, D. Estancamiento, desequilibrios y ruptura: 1955-1972. En: Instituto de Economía. El Uruguay del siglo XX: la economía. Montevideo: Ediciones de la Banda Oriental, 2005. p.65-94.

BRALICH, J. La formación universitaria de asistentes sociales: más de seis décadas de historia. Cuadernos de Trabajo Social, n.2, p.9-48, 1993.

CAETANO, G; RILLA, J. Historia contemporánea del Uruguay: de la Colonia al MERCOSUR. Montevideo: Fin de siglo, 1996.

CASTEL, R. Las metamorfosis de la cuestión social: una crónica del salariado. Buenos Aires: Paidós, 2004.

CASTELLANOS, E. Uruguay, un caso de bienestar de partidos. Revista Uruguaya de Ciencia Política, n.9, 1996.

D’ELIA, G. E1 Uruguay neobatllista: 1946-1958. Montevideo. EBO, 1982.

DE SWAAN, A. A cargo del Estado. Barcelona: Ed. Pomares Corredor, 1992.

DOUGLAS, M. Cómo piensan las instituciones. Madrid: Alianza Editorial, 1996.

FILGUEIRA, C.; FILGUEIRA, F. El largo adiós al país modelo: políticas sociales y pobreza en el Uruguay. Montevideo: Kellogg Institute, 1994.

FINCH, H. Historia económica del Uruguay contemporáneo. Montevideo: EBO, 1980.

Redefinición de la utopía en Uruguay: la política de bienestar social posterior

a 1940. Revista Uruguaya de Ciencias Sociales. 2.serie, Año 14, p.7-20, 1989. 
FOUCAULT, M. Historia de la sexualidad: la voluntad de saber. México: Ed. Siglo XXI, 1998.

. Vigilar y castigar: nacimiento de la prisión.. 1.ed.; 3.reimpr. Buenos Aires, Siglo XXI, 2004.

Seguridad, territorio, población. Curso en el Collège de France: 19771978. Buenos Aires. Fondo de Cultura Económica, 2006.

HACKING, I. Philosophie et histoire des concepts scientifiques. Leçon inaugurale faite le jeudi 11 janvier 2001 par Ian Hacking. Disponible en: < http:/ /www. college-de-france.fr/media/pub-lec/UPL52662-LI-157-hacking.pdf $>$. Consulta en: 9 novembro 2008.

HOBSBAWM, E. Historia del siglo XX. Buenos Aires: Crítica, 2007.

KRUSE, H. Evolución de los modelos de ayuda en el Uruguay. Revista Trabajo Social, Uruguay. Montevideo, n.2, p.18-23, 1987.

En procura de nuestras raíces. Cuadernos de Trabajo Social, Montevideo. FCS. DTS. UDELAR, n.3, p.87-106, 1994.

MIDAGLIA, C; ANTÍA, F. La izquierda en el gobierno: ¿cambio o continuidad en las políticas de bienestar social? Revista Uruguaya de Ciencia Política, ICP. Montevideo, n.16, 2007.

MITJAVILA, M; SILVA, C. Globalizaçao, modernidade e individualizaçao social. Revista Katálysis, v.7, n.1, UFSC, 2004.

MORÁS, L. E. De la tierra purpúrea al laboratorio social. Montevideo: Ediciones de la Banda Oriental, 2000.

ORTEGA, E. El servicio social y los procesos de medicalización de la sociedad uruguaya en el período neobatllista. Montevideo: Trilce, 2008.

Medicina, religión y gestión de lo social: un análisis genealógico de las transformaciones del Servicio Social en el Uruguay - 1955-1973. Tesis (Doctorado en Ciencias Sociales con especialización en Trabajo Social), Facultad de Ciencias Sociales, Universidad de la República, Uruguay, 2011. En prensa.

ORTEGA, E; VECINDAY, L. De las estrategias preventivistas a la gestión del riesgo: notas sobre los procesos de individualización social. Revista Fronteras, FCS-DTS. n.5, Segunda Epoca, 2009.

SERNA, M. Las políticas de la pobreza en los gobiernos de izquierda del cono sur: de las redes sociales de seguridad a las redes de protección social. En: LIMA, María Regina Soares de (Org.). Desempenho de governos progressistas no Cone Sul: agendas alternativas ao neoliberalismo. Rio de Janeiro: Ed. IUPERG, 2008.

WEISSHAUPT, J. R. (Org.). As funçoes sócio-institucionais do Serviço Social. São Paulo: Cortez, 1988. 